The $8^{\text {th }}$ International Conference of the Slovenian Society for Non-Destructive Testing

"Application of Contemporary Non-Destructive Testing in Engineering"

September 1-3, 2005, Portorož, Slovenia, pp. 11-17

\title{
STUDY OF PHASED ARRAY TECHNIQUES FOR CONCRETE INSPECTION
}

\author{
O. Paris ${ }^{1}$, C. Poidevin ${ }^{1}$, J.M. Rambach ${ }^{2}$, G. Nahas ${ }^{2}$ \\ ${ }^{1}$ CEA, Commissariat à l'Energie Atomique, SYSSC/LMUS \\ 91191 Gif sur yvette cedex, France, E-mail: olivia.paris@cea.fr \\ ${ }^{2}$ IRSN, Institut de Radioprotection et de Sûreté Nucléaire, IRSN, France
}

\begin{abstract}
From a safety standpoint the concrete containment of the nuclear power plant is one of the most essential components because it serves at the final barrier to the release of fission products. The safety authority supervises the study and development of a phased array equipment for ultrasonic testing of these concrete structures. The aim is to detect and characterize the presence of cracks which can be responsible for loss of structural capacity or of leak tight integrity of the containment. Typical frequency range used for ultrasonic testing of concrete is between 50 and $300 \mathrm{kHz}$ which provides a wide and non directive ultrasonic beam leading to resultant poor signal to noise ratios. In order to improve the performances of inspection, we are studying specific techniques of reconstruction such as SAFT processing and phased array method.

One objective is to use the focusing and steering of the ultrasonic beam in order to reconstruct trough imaging tools the whole profile of a detected defect. First measurements and reconstructions performed on an artificial crack embedded in a concrete block will be presented. Propositions made to improve the existing system are discussed trough simulations of the ultrasonic field.
\end{abstract}

Keywords: Ultrasonic testing of concrete, Phased array techniques, SAFT processing

\section{Introduction}

Wall containments in Pressurized Water Reactor are extremely important because they must retain radioactive materials if an accident should occur. These walls are thick $(1.2 \mathrm{~m}$ or $0.9 \mathrm{~m})$ pre-stressed and reinforced concrete structures containing pretensionned cables. The containment is cylindrical with an hemispherical dome. For the safety authority (IRSN) it is essential to maintain the integrity of the wall containment of nuclear power plant.

The concrete containment building of nuclear power plant structures are susceptible to ageing by various processes depending on the operating environment and service conditions. In order to ensure the structural capacity and leak tightness of the concrete structure it is necessary to detect cracking phenomena which is one of the primary sign of ageing. Potential degradations of the concrete material can affect the ability of a nuclear power plant containment to perform satisfactorily its functions. The degradation of concrete can be the result of various attacks [1]. Physical attack mechanisms include freeze/thaw cycling, thermal expansion, thermal cycling 
abrasion. Chemical attacks may also occur in several forms : efflorescence, attack by sulphate and by acids, salt crystallization and alkali-aggregates reaction.

Such mechanisms and fatigue effects can be responsible for surfaces and bulk flaws such as cracks, porosity and laminations.

The aim of our work, supported by IRSN, is to detect non destructively and to determine the crack shape inside the concrete wall and to check the defect growth between two inspections. Ultrasonics were identified as having a great potential to inspect such thick concrete structures.

Compared to inspection of steel components the difficulty of ultrasonic control in concrete comes from the heterogeneous structure. Concrete is a composite material constituted of a binding cement medium with embedded particles from sand to gravel with size up to 20-25 mm. The propagation of a the wave in such media generates heavy scattering and attenuation of the sound energy that provide a resultant poor signal to noise ratio (SNR) of the reflected signal amplitudes.

For that reason typical frequency range used for ultrasonic control in concrete is quite low between 50 and $300 \mathrm{kHz}$. In order to increase the SNR we propose ultrasonic techniques inspired from SAFT processing and phased array methods using a combination of several transducers.

Mock up calibration blocks were made in order to develop test procedures. One sample includes a known artificial crack composed of different orientated facets localised at different depth.

\section{Samples}

The formulation of concrete used for that study is well representative to the one used in wall containment with a maximum size of aggregates of $25 \mathrm{~mm}$. Early experimental measurements have been carried out on a concrete block of 800x800x600 $\mathrm{mm}^{3}$

An artificial crack composed of four facets with different orientations has been included in the block. Each facet noted Pi on Fig. 1 has a surface of $40 \times 70 \mathrm{~mm}^{2}$ and is considered as a reflector for sound wave. These facets are supposed to represent the surface extension of crack, they are located between $200 \mathrm{~mm}$ and $500 \mathrm{~mm}$ below the surface of inspection and tilted between $10^{\circ}$ and $20^{\circ}$. We assume that crack with an aperture of $100 \mu \mathrm{m}$ acts as a perfect reflector for ultrasonic waves propagating in concrete.

a) Inspection surface

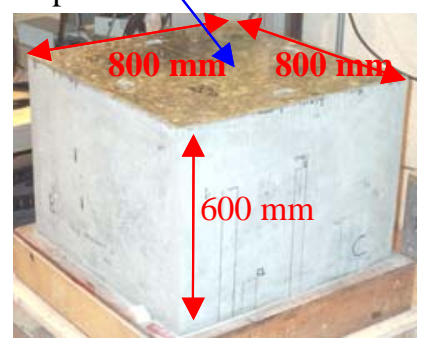

b) Flaw description
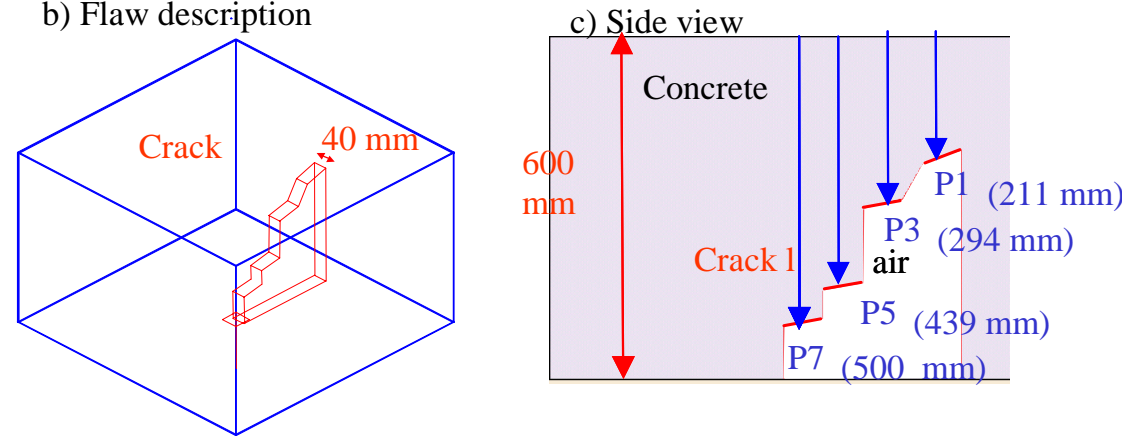

Fig. 1: a) Sample of concrete b) 3D view of that sample c) Crack with the different facets "Pi" to be detected. 


\section{Ultrasonic equipment tested}

The pulse echo technique is commonly used to detect and characterize cracks in steel components. Such ultrasonic inspection is based on the analysis of the backscattered signal from the inside of the concrete which is collected during the acquisition.

As we try to detect with an increased spatial resolution small reflectors in a medium with aggregates of $20 \mathrm{~mm}$, we use broadband transducers (diameter $38 \mathrm{~mm}$ ) operating at $250 \mathrm{kHz}$. In order to improve the signal to noise we use a set of six probes to perform signal reconstruction. Transducers are mechanically displaced in order to scan the surface of the concrete.

A multi channel system is used which allows to drive the 6 transducers used, to adjust their relative parameters (delay at transmitter or receivers, amplitude..). The signal of excitation is $250 \mathrm{~V}$ square pulse. The coupling is a film of water and the probes are spring loaded in order to compensate the variations at the surface of the block due to possible roughness .

\section{Study of different ultrasonic techniques}

Two techniques of reconstruction have been applied to experimental measurements in order to improve the detection of the artificial crack. Their principle is briefly presented and results are discussed.

\subsection{Multi-probes reconstruction}

In order to improve the signal to noise ratio of the measurements we apply the principle of reconstruction used in SAFT processing [2] with an arrangement of transducers. The set of transducers is displaced mechanically over the surface of the concrete to be scanned. One probe is used as an transmitter while others are functioning as receivers. After the acquisition, the reconstruction consists in a combination of the recorded signal for each channel and position in scan axis after a fit in time and position (Fig. 3). Such a fit takes into account the delay of the reflected wave introduced by the transmitter-receiver distance. This reconstruction must have a constructive effect on the echo of flaw and a destructive one on the noise which leads to an improvement of the signal to noise ratio.

We apply this procedure to detect the different facets of the artificial crack. The reconstructions are made on Bscan images which represent the signal along the scan axis versus depth. The transducers are disposed as shown in Fig. 2, the smallest distance probe to probe is $80 \mathrm{~mm}$. We present the result of reconstruction from 10 raw Bscans. The Bscan image resulting from a single transducer in pulse echo mode is compared to the reconstructed results.

The variations of color indicate areas of different acoustical response either due to structural noise or to the presence of flaw. We observe on results presented in Fig. 2 an important structural noise in mono element case which is importantly reduced in the reconstructed case. The reconstruction is successful since facets $\mathrm{P} 1$ and $\mathrm{P} 3$ are clearly detected by applying multi-probes reconstruction.

\subsection{Phased array techniques}

In order to apply phased array technique to concrete we also use an array of several transducers. The electronic focusing produced by a large aperture array of transducers should improve sensitivity and signal to noise ratio. The focusing of the ultrasonic beam is performed by applying delay laws on transducers at transmission and reception. This enhances and concentrates the energy at a selected depth which increases the resolution and signal to noise ratio. Preliminary measurements in transmission [3] have successively shown the ability of focusing an ultrasonic beam in a concrete structure. Compared to multi probes reconstruction phased array technique takes the advantage of a concentrate focal spot transmitting higher energy on a selected region. 

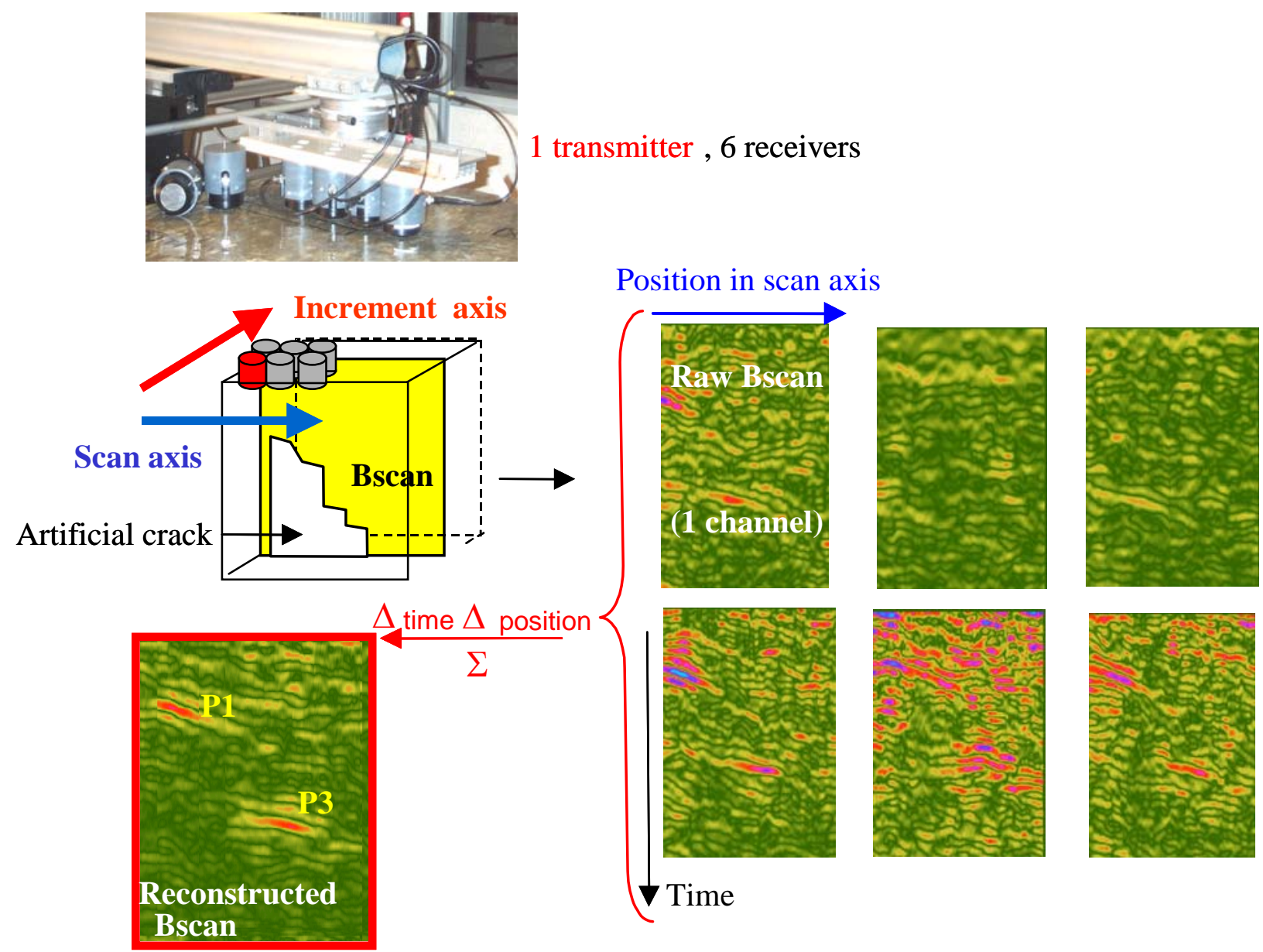

Fig. 2: Principle of multi-probes reconstruction in reception.

\subsection{Comparison of multi-probes reconstruction and phased array techniques}

We apply phase array technique to the detection of the crack profile with the same arrangement of transducers than the one used for multi-probes reconstruction study. The focusing is done by adjusting four delay laws in order to steer the ultrasonic beam perpendicularly to each facets to be detected.

The Bscans images resulting from the application of the two studied techniques are compared in Fig. 3 to a Bscan measured with in mono-element. The profile of the crack is plotted in dotted line on the Bscan in order to compare relative positions of the facets and to detected echos.

Both techniques lead to efficient reconstructions, the structural noise is importantly reduced compared to the mono-element case and there is a good match in time and position of the theoretical profile of the crack with the detected echoes coming from the different reflectors.

Multi-probes reconstruction improves the detection of facets near the surface of inspection but for deep facets the signal to noise ratio remain weak. The fact the energy is transmitted only by one transducer limits the results deeper depth (after P3).

Tests of focussing the ultrasonic beam have also been performed successfully at different depths of the crack. The steering of the ultrasonic beam adapted to each tilted facets optimizes the direction of the reflected energy toward the receivers. This improves the detection of the crack compared to a control without deflecting the ultrasonic beam [4].

\section{4 'Tomo-SAFT': a reconstruction technique based on angular scanning}

An other proposed technique consists to apply angular scanning by using different delay laws to transducers. Then a post acquisition reconstruction is carried out on measurements using same 
principle as in multi-probe reconstruction (part 2.1). Each raw measurement corresponding to one angle of inspection is summed constructively to each others. Such procedure enhances the flaw echo and reduce the structural noise.
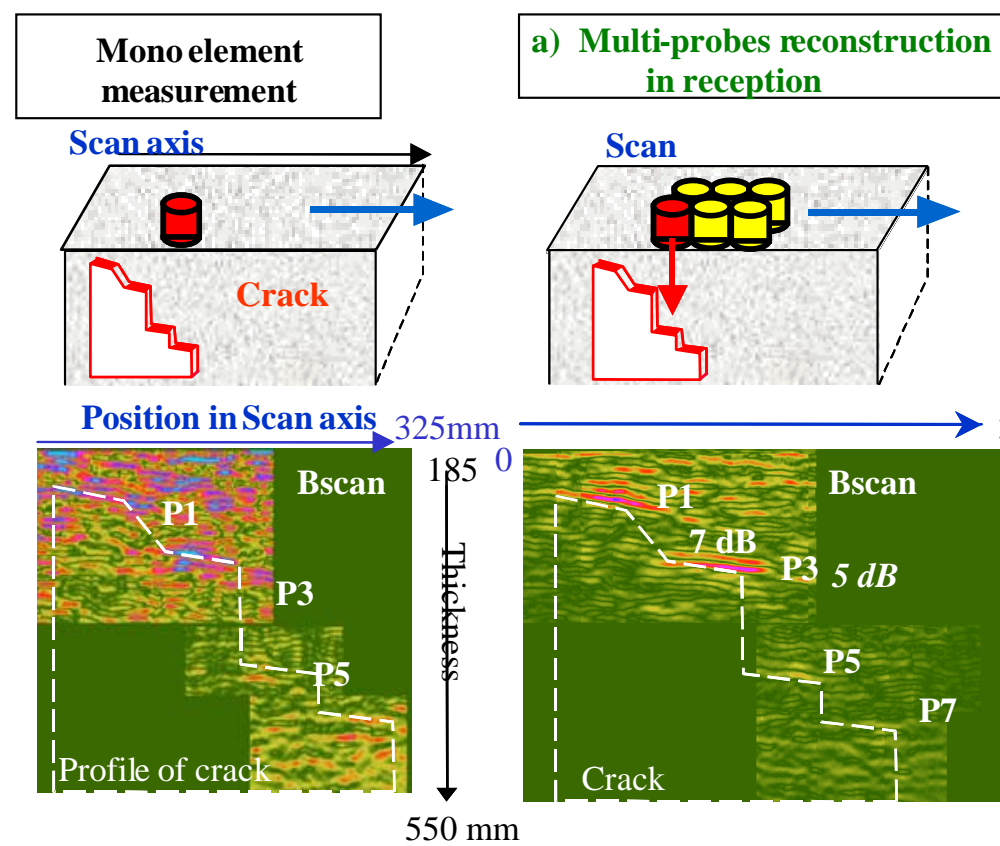
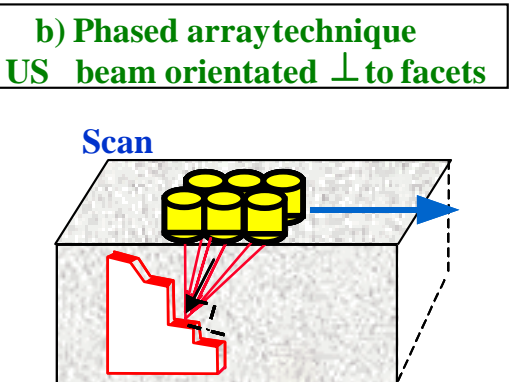

$375 \mathrm{~mm}$

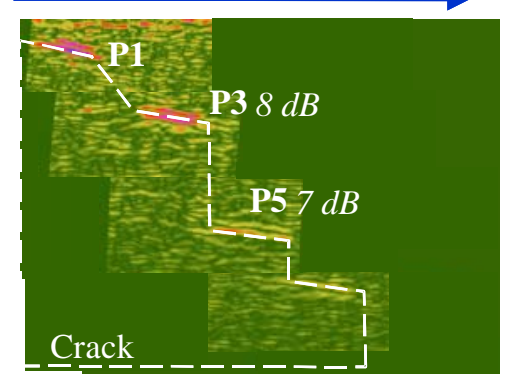

$535 \mathrm{~mm}$

Fig. 3: Comparisons between mono-element measurement of the crack and the reconstructed Bscans images resulting from multi-probes and phased array measurements.
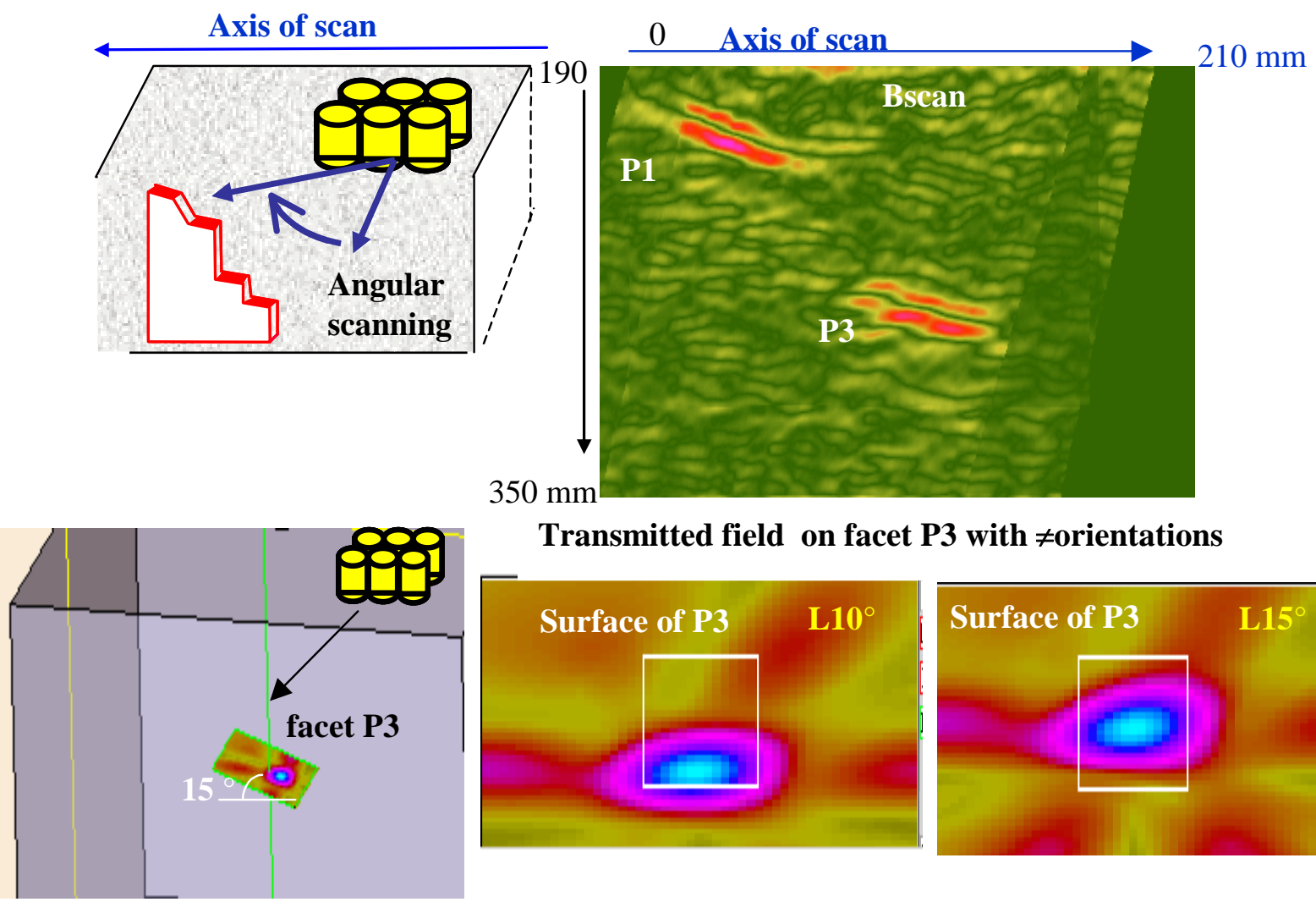

$350 \mathrm{~mm}$

Transmitted field on facet $P 3$ with $\neq$ orientations
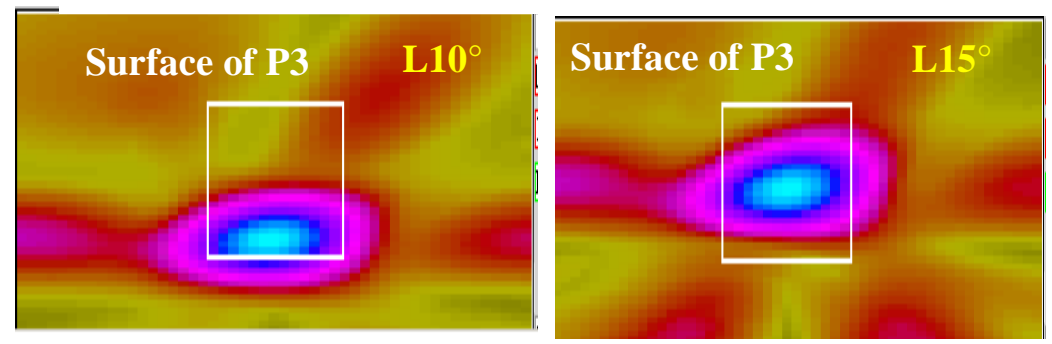

Fig. 4: Results of a third proposed technique based on angular scanning and post acquisition reconstruction. 
Such technique takes advantage of phase array technique since the maximum energy is transmitted at a given depth and of post acquisition reconstruction that improves SNR by averaging the structural noise over the whole aperture of the transducer.

The principle of this technique could be adapted to blind inspections as the application of various angles of inspection is favorable to the detection of defect which orientation is unknown.

The principle of the method is illustrated in Fig. 4.

We present simulations of the ultrasonic field performed with CIVA Software. Such simulations indicate the angles of inspection to be used for reconstructing a given defect. For example of facet P3 $15^{\circ}$ tilted, we observe on simulations that for angles lower than $10^{\circ}$ the maximum transmitted energy is coming out from the surface of the facet and should inefficiently contribute to reconstruction. According to simulations reconstruction of P3 should be optimizes for an angular scanning between $10^{\circ}$ to $20^{\circ}$. In Fig. 4 is shown the reconstruction of P3 for three angles of inspection which are $10^{\circ}, 13^{\circ}$ and $15^{\circ}$.

\section{Improvement of instrumentation}

The experimental measurements have shown the concrete acts a filter for the higher frequencies $(>200 \mathrm{kHz}$ ). So it would be useful to choose a lower central frequency or to widen the bandwith to ensure a better resolution of the method. We also propose to reduce the size of each transducer in order to improve the focusing in phased array techniques. This should enhance the transmitted energy and reduce the sides lobes.

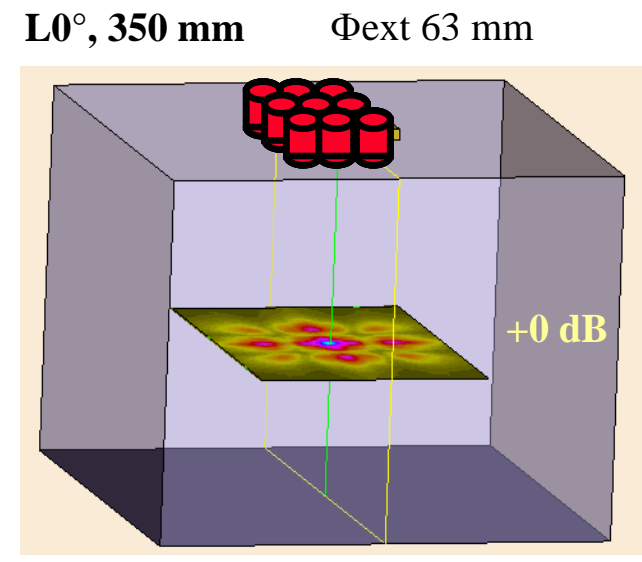

Фext $45 \mathrm{~mm}$

$\mathrm{L}^{\circ} 0^{\circ}, 350 \mathrm{~mm}$

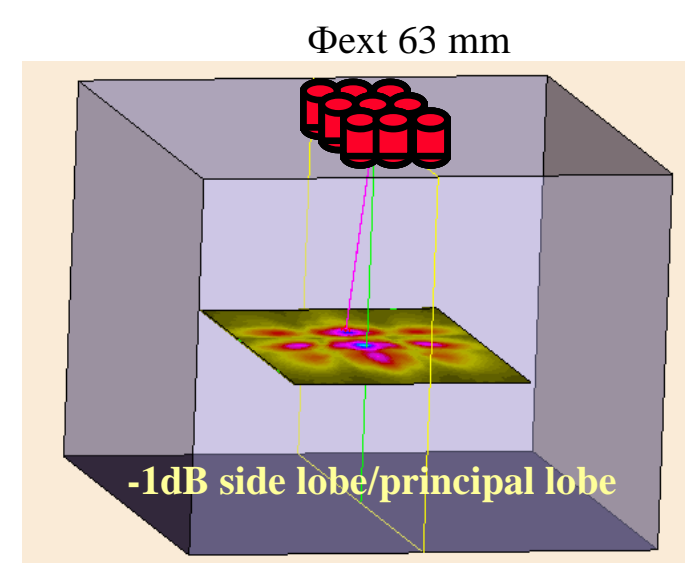

Aperture 170x170mm²

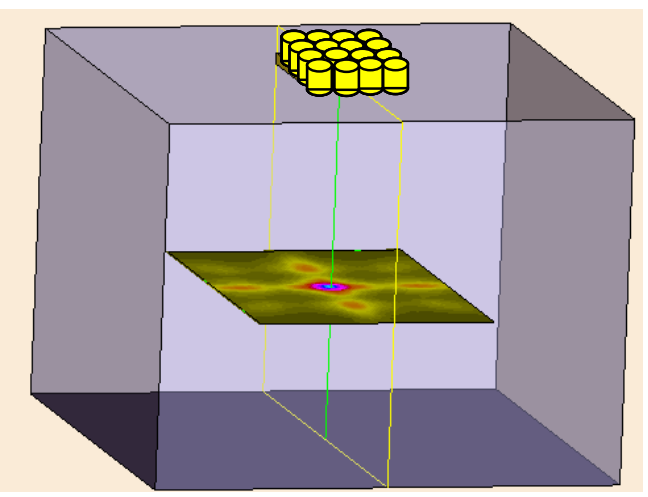

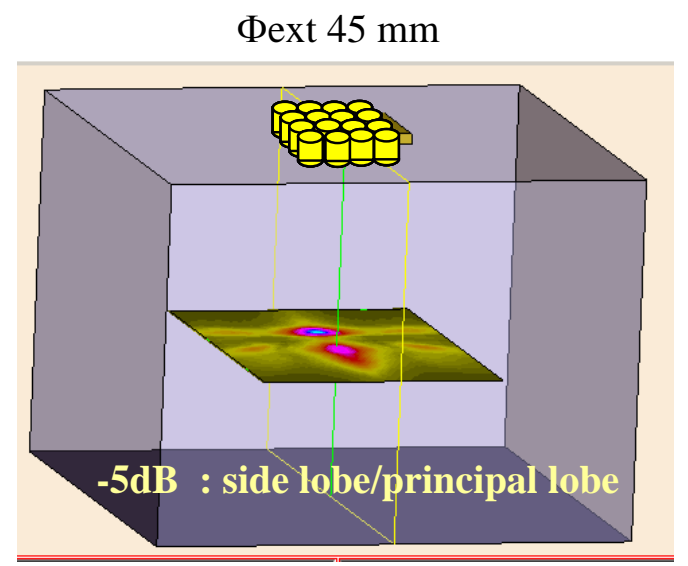

Fig. 5: Transmitted ultrasonic fields generated by 2 arrays of transducer in normal incidence $0^{\circ}$ and at $20^{\circ}$ at $350 \mathrm{~mm}$ deep. 
Fig. 5 presents the ultrasonic fields generated by an array of $170 * 170 \mathrm{~mm}^{2}$ composed of 9 transducers identical to those used in the previous study (Фexternal 63mm, Фpiezo 38mm). An array of same aperture composed of 16 transducers with higher bandwidth and reduced diameter (Фexternal 45mm, $\Phi$ piezo $34 \mathrm{~mm}$ ) is also studied. The transmitted fields are calculated on plane parallel to the surface of inspection at $350 \mathrm{~mm}$ deep in an normal orientation ( $\mathrm{L} 0^{\circ}$ ) of the ultrasonic beam and at $20^{\circ}$.

The new characteristics of transducer should improve the performances of the array because : the transmitted amplitude is enhanced of about $5 \mathrm{~dB}$ compared to the transducer firstly used and the contribution of sides lobes is also importantly reduced.

Additional experiments must be perform to validate the new choice of transducer and to achieve the realization of the ultrasonic equipment dedicated to concrete inspection.

\section{Conclusions}

The aim of that study is to characterize non destructively cracks in concrete wall containment of power nuclear plant. Several ultrasonic techniques of reconstruction have been developed and applied to the detection of an artificial crack embedded in a concrete lab block.

Early results have shown the great potential of such techniques. Multi probes reconstruction using principle of SAFT processing have been tested with a set of several transducers. The reconstruction using theoretical parameters of reconstruction is effective to detect with an improved signal to noise ratio the less depthless facets composing the crack. Phased array technique also improves performance of the method in term of signal to noise ratios and sensibility by optimizing the transmitted energy.

Improvements of the instrumentation have also been studied through ultrasonic field simulations; The final multi-channel ultrasonic equipment should allow to apply any reconstruction technique and provide full reconstructed images of the concrete structure which show the profile of flaws. In parallel to further experimental validations, we will develop tools in order to simulate more realistically ultrasonic control in concrete; This needs to take into account the heterogeneous media and to include in CIVA models the attenuation and prediction of the structural noise of concrete.

\section{References}

[1] T. M. Refai : Review of NPP concrete degradation factors and assessment methods, Processing of structures congress, Texas, 1992.

[2] M. Shickert : Towards SAFT imaging in ultrasonic inspection of concrete, International Symposium Non Destructive Testing in Civil Engineering (NDT-CE) 411-148 September 1995.

[3] S. Mahaut, G. Cattiaux, O. Roy and Ph. Benoist : Improvement of defect characterization with ultrasonic adaptive focusing technique, 14th Int. Conf. on NDE in the Nucl. and Pres. Ves. Ind., p. $427,1996$.

[4] O. Paris, Ph. Brédif, O. Roy : Study of phased array techniques for cracks characterization in concrete structures, International Symposium Non Destructive Testing in Civil Engineering (NDT-CE) 411-148 September 2003. 